\title{
Comparison the Theoretical and Experimental Results of Radio Absorbing Properties of Au Thin Films in 8-11.6 GHz Band
}

\author{
Oleksandr Bediukh and Maksim Drobkov \\ The Department of Quantum Radiophysics, Faculty of Radio Physics, Electronics and Computer Systems, Taras Shevchenko \\ National University of Kyiv, 60 Volodymyrska Street, Kyiv 01033, Ukraine
}

\begin{abstract}
The comparison of theoretical and experimental results of measurements of absorbing properties of Au films of $10 \mathrm{~nm}$ thickness deposited on a polymer substrate in the frequency band 8-11.6 GHz electromagnetic radiation are presented. The new configuration of $\mathrm{Au}$ thin film in a rectangular waveguide had been considered. An abnormally high level of absorption of electromagnetic radiation throughout the range of wavelengths was obtained. This is apparently due to the lack of galvanic contact between the film and waveguide.
\end{abstract}

Key words: Radar absorbing materials, nano-scale Au films, VSWR, 3-cm wavelength range.

\section{Introduction}

The development of new, improved RAM (radar absorbing materials) is one of the main components of the task of protection from EMR (along with screening, the use of design elements, etc.).

There are resonant absorbers, which are one type of RAM [1]. They use semitransparent metal films. If such film is situated at a distance of $\lambda / 4$ ( $\lambda$-wavelength of electromagnetic radiation) before conductive surface which has to be protected, it is provided the conditions for optimal matching at this wavelength. When several films use, with help of modern computational methods, it is possible to optimize not only the absorption band, but and number of layers, the total thickness and weight of the RAM.

Additional studies are conducted and for studying of the absorbing properties of metal films [2, 3]. These works are carried out on the assumption that the film thickness is much smaller than the thickness of the skin

Corresponding author: Oleksandr Bediukh, senior research associate, Ph.D., research field: radio spectrum management. E-mail: bedjukh@univ.kiev.ua. layer in the selected band of wavelengths. At a film thickness of 10-100 nm, this assumption holds for the microwave range.

The results of measurement of reflection and absorption of electromagnetic waves of the Au films in the frequency band 25-37 GHz and 8-12 GHz are presented in Ref. [4] and Ref. [5], respectively.

In our paper we compare the theoretical and experimental results of measurements of absorbing properties of Au films of $10 \mathrm{~nm}$ thickness deposited on a polymer substrate in the frequency band $8-11.6 \mathrm{GHz}$ electromagnetic radiation. The paper is organized as follows: Section 2 shows the experimental technique; Section 3 presents the comparison of experimental results with calculated data; Section 4 gives conclusions.

\section{Experimental Technique}

For research it is used a gold (Au) film of $10 \mathrm{~nm}$ thickness, obtained by thermal vacuum deposition on a dielectric polymer substrate of $0.1 \mathrm{~mm}$ thickness. Control the thickness of the film is implemented by known method of quartz balance. 
The samples were cut with size slightly smaller than the cross section of the waveguide so that they are easily placed in him. The sample was placed between the layers of foam as shown in Fig. 1. The Au film had no galvanic contact with the walls of the waveguide.

Measurements were carried out in the frequency band 8-11.6 GHz (3-cm wavelength range) using of a VSWR meter panoramic P2-65 (similar to a scalar network analyzer) in the section of rectangular waveguide with a cross section of $28 \times 12 \mathrm{~mm}$ for two cases: the segment of the waveguide was joined to the shorting in the form of metal plate or to the matched load.

\section{The Comparison of Experimental Results with Calculated Data}

For comparison, we choose the result of measurements of the best indicators for absorption. These results are consistent with film thickness $10 \mathrm{~nm}$ and the distance from the film to the shorting equal to $1.5 \mathrm{~mm}$ (see Fig. 1 from Ref. [5]).

The power reflection coefficient $R$ from the measured of $V S W R$ is calculated as Eq. (1):

$$
R=\frac{(V S W R-1)^{2}}{(V S W R+1)^{2}}
$$

The absorption coefficient $A$ can be written as Eq. (2):

$$
A=1-R
$$

Relative dielectric constant of the foam is about 1.1 and it of the polymer used for the substrate is about 2 .

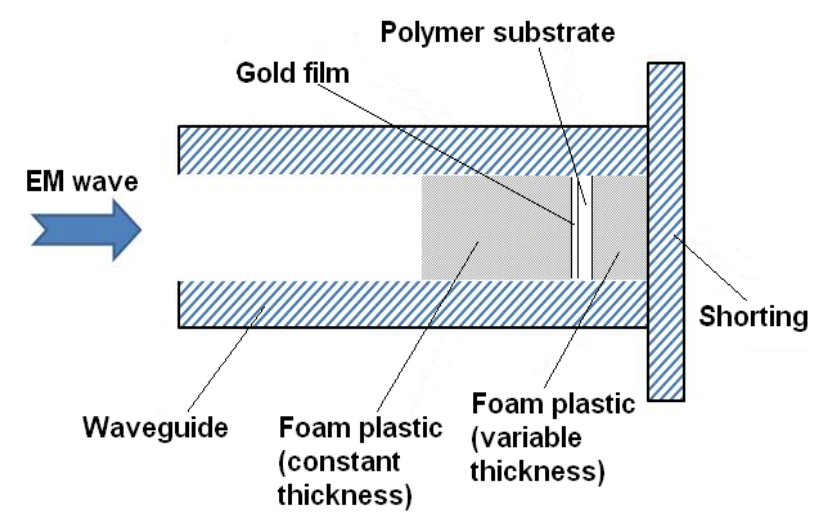

Fig. 1 The placement of the samples in a segment of the waveguide line connected to a shorting.
Moreover, the dielectric loss tangents of both of these materials are less than $10^{-3}$.

In view of this, and the fact that the thickness of the polymer substrate is $0.1 \mathrm{~mm}$, which is about $1 / 300$ of the wavelength in the waveguide in the band measurements, we neglect reflections of EMR (electromagnetic radiation) which associated with the presence of both the foam and the polymer substrate.

We assume that the Au film is in air-filled waveguide. The thickness of the skin layer in the selected band of frequency for gold is more than an order greater than the thickness of the film.

In this case, the EMR penetrates the entire thickness of the film and the reflection coefficient $G_{1}$ for the film having a thickness $d$ and a conductivity $\sigma$ can be written as Eq. (3) [2]:

$$
G_{1}=-\beta /(1+\beta)
$$

where, $\beta=\sigma d /\left(\varepsilon_{0} c\right), \varepsilon_{0}-$ the permittivity of vacuum, $c$ - the speed of light in vacuum.

Accordingly, the transmission coefficient is equal to:

$$
T_{1}=1+G_{1}=1 /(1+\beta)
$$

At a film thickness of $10 \mathrm{~nm}$ conductivity $\sigma$ depends both on the thickness and on the method of preparation of the film. On the other hand, in the case of connecting to the matched load, $G_{1}{ }^{2}$ corresponds to the power reflection coefficient that is given as Eq. (1). Therefore, to determine the value of $\beta$, we use the experimental values of the power reflection coefficient for the case of the matched load.

From the measurement results, which are shown in Fig. 2a from Ref. [5], we see that the value of $V S W R$ for the case of 10 -nm gold film does not change with the frequency and is equal to about 4.5 .

Using the Eq. (1) we have $R \approx 0.4$ and using Eq. (3) we get $\beta \approx 1.72$.

The reflection coefficient of the field $G$ for the case of shorting connection, taking into account the multiple reflections between the gold film and the reflecting surface of shorting, can be written as Eq. (5):

$$
G=G_{1}-\frac{T_{1}^{2} e^{-i 4 \pi h / \lambda_{0}}}{1+G_{1} e^{-i 4 \pi h / \lambda_{0}}}
$$




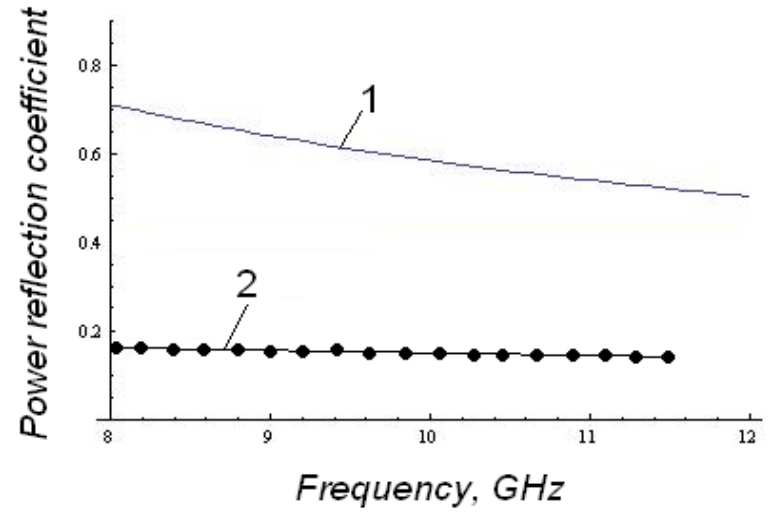

Fig. 2 Experimental (curve 2 with circles) and theoretical (curve 1) dependence of the power reflection coefficient from frequency.

where, $\lambda_{0}=\lambda / \sqrt{1-(\lambda / 2 a)^{2}}$ - the wavelength in the waveguide, $a$-the size of the wide wall of the waveguide.

The power reflection coefficient $R$ is equal to square of modulus of the complex reflection coefficient $G$ :

$$
R=|G|^{2}
$$

Theoretical values of $R$ as a function of frequency, calculated on the basis of Eqs. (5) and (6) for $\beta=1.72$ are shown in Fig. 2.

As seen in Fig. 1, the calculations do not give satisfactory agreement with the experimental results. The theoretical power reflection coefficients (curves 1) are in 3-5 times greater than those obtained from the experiment (curve 2). In addition for the theoretical curve it is observed a pronounced dependence from the frequency. The theoretical reflection coefficient decreases with frequency increasing what corresponds to the expected reduction of reflection when we approach to the theoretical minimum at the frequency corresponding to $\lambda_{0} / 4$ of the distance between the gold film and the reflecting surface of the shorting.

\section{Conclusions}

Experimental data of the power reflection coefficient obtained for the $\mathrm{Au}$ film thickness of $10 \mathrm{~nm}$ at a distance $1 \mathrm{~mm}$ from the reflecting surface of the shorting were significantly less than that were calculated according to traditional theory in the frequency band 8-11.6 GHz.

The absorption is fixed on a level more than $80 \%$ and almost not depended from frequency that can significantly expand the ability to use such films in practical radar absorbing materials.

An abnormally high level of absorption of electromagnetic radiation throughout the range of wavelengths was apparently connected with the lack of galvanic contact between the film and waveguide. Absence of the contact leads to an additional reactance, resulting in a phase change of electromagnetic radiation.

We plan to conduct further experimental and theoretical studies of this effect and present the results in the following article.

\section{References}

[1] Saville, P. 2005. Review of Radar Absorbing Materials. Technical Memorandum, DRDC Atlantic, TM 2005-003.

[2] Andreev, V. G., Vdovin V. A., and Voronov P. S. 2003. "An Experimental Study of Millimeter Wave Absorption in Thin Metal Films.” Technical Physics Letters 29 (2003): 953-5.

[3] Antonets, I. V., Kotov, L. N., Nekipelov, S. V., and Karpushov, E. N. 2004. "Conductive and Reflective Properties of Thin Metal Films.” Technical Physics 49 (Nov.): 1496-500.

[4] Bediukh, O., and Tepliy, O., 2012. "Investigation of Radio Absorbing Properties of Thin Metal Films in the Microwave Range.” Presented at the XII International Young Scientists' Conference on Applied Physics, Kyiv, Ukraine.

[5] Bediukh, O., Drobkov, M. 2013. "Investigation of Radio Absorbing Properties of Thin Metal in 8-11.6 Ghz Band.” Presented at XIII International Young Scientists' Conference on Applied Physics, Kyiv, Ukraine. 